\author{
Military Technical College \\ Kobry El-Kobbah, \\ Cairo, Egypt.
}

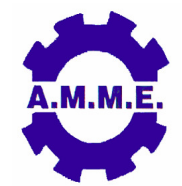

$15^{\text {th }}$ International Conference on Applied Mechanics and Mechanical Engineering.

\title{
EXPERIMENTAL AND NUMERICAL INVESTIGATION OF THE CAPABILITIES OF PERIODIC PLATES FOR VIBRATION ATTENUATION
}

\author{
S. Talaat ${ }^{1}$, A. Desoki ${ }^{2}$, M. Tawfik ${ }^{2}$ and H. M. Negm ${ }^{2}$
}

\begin{abstract}
In the previous decades, many numerical and experimental investigations on the capabilities of periodic structures for vibration attenuation and localization were performed and proved those capabilities for different simple structures. Earlier investigation of such phenomenon in periodic plates was not promising due to different factors. In this research, experimental and numerical investigations of the vibration response of different periodic plates are investigated to examine similar behavior of periodic plates. Three different periodic configurations were tested under 'almost' free support boundary conditions. This work is an important over that performed by Hull [9]. Milled plates are used to avoid the effect of the adhesive material in damping which was used [3]. The experiments involved the excitation from one corner of the square plate and measuring the response at the three other corners. The results were compared to that of a similar size plain plate. The results indicated the reduction of the response of the periodic plates, which have overall less weight, by ratios that may exceed $10 \mathrm{db}$ in different frequency bands. The results of this work, verified by experiments, encourage more investigation on the localization capabilities of those plates as well as the preparation of numerical models that may predict those results in a more accurate fashion.
\end{abstract}

1 Space Division, National Authority of Remote Sensing and Space Sciences, Cairo, 11769, Egypt.

2 Aerospace engineering Department, Cairo University, Giza, 12613, Egypt. 


\section{NOMENCLATURE}

$\mu \quad$ Propagation factor.

$\alpha \quad$ the attenuation in the vibration amplitude between input and output.

$\beta \quad$ Phase difference between the input and the output vibration waves.

\section{INTRODUCTION}

Over the last decades, research on effective methods for attenuating vibration levels in structures has been very active. The periodic structure technique is one of these effective methods which have a lot of applications especially for the aerospace industry such as satellite solar panels, aircraft fuselages and wings. A periodic structure consists fundamentally of a number of identical structural components which are joined together to form the whole structure. Discontinuities which can be induced with various forms such as masses, cross sectional geometries, and changes in material, can cause reflections of the vibration wave that may reduce the transmitted energy. Yet the degree of attenuation is insufficient or inadequate over a broad range of frequencies and the regions between these discontinuities exhibit local resonances.

The existence of complete band gaps and resonances in a plate with a periodic stubbed surface was demonstrated numerically and experimentally [1]. It was demonstrated that as the stub height approaches about triple the plate thickness a complete narrow band gap develops. The vibration control of a cantilever aluminum plate using a periodic configuration of $\mathrm{RL}$ (resistive-inductive) shunted piezoelectric patches was investigated in [2]. Numerical and experimental analyses were performed also to 4-node Kirchhoff plate elements and a reverse approach was used for the finite element analysis. Unit cell analysis showed that increasing the shunting resistance reduces the attenuation but increases the region of effectiveness. Experimental analyses demonstrated that the tuning capabilities of the $R L$ networks can be effectively used within a periodic framework to obtain a broadband control effect. Both numerical and experimental results were encouraging in the sense that a unit cell approach is capable of accurately predict the actual response of the system.

Also the vibration characteristics of a plate with shunted piezoelectric patches were also investigated by Ref. [3]. The response was investigated when the patches were periodically distributed. He formulated a plate spectral finite element model and compared the results with experimental results, which showed reasonable agreement. Later numerical and experimental investigations of the application of a periodic array of resistive-inductive $(R L)$ shunted piezoelectric patches for the attenuation of broadband noise radiated by a flexible plate in an enclosed cavity was investigated [4]. That research showed that the tuning capabilities of simple RL shunt circuits were combined with filtering characteristics of periodic structures to obtain a tunable periodic plate, and the resultant smart structure experienced significant reductions in its noise radiation capability. Numerical simulations demonstrated the applicability of Bloch theorem [5] for unit-cell analysis on the in vacuo plate [6] as a tool to predict the frequency ranges of effective structural acoustic control. Also, experiments were performed to validate the numerical predictions, and to demonstrate the effectiveness of the proposed strategy. 
Recently a thin epoxy plate containing a periodic square array of lead discs hemmed around by rubber was considered [7]. The band structures were calculated theoretically using a periodic expansion by means of Bloch theorem. The numerical results showed that the full band gaps of flexural vibration do exist in the thin plate. It was also shown that the width of the first full band gap is about three times larger than that in a thin steel plate with 2D binary locally resonant structures. The effect of lattice constant [7] and thickness of the plate on the band gap have also been demonstrated. Casimir and Kevorkian [8] presented the principle of developing two dimensional elements such as plates and they showed how symbolic computation software could be used in this purpose. The method of construction of dynamic stiffness matrices was applied to the rectangular Kirchhoff continuous elements, and a study of harmonic response over a large frequency range was made. It offers a clear advantage over finite elements, particularly its high precision and low memory cost. In Ref. [8] the effect of periodicity on the frequency response of different forms of periodicity was also investigated.

An analytical model that incorporates an infinite number of periodically spaced masses into the equations of elasticity that model motion and stress in a twodimensional fully elastic solid was studied by Hull [9]. The examination of a periodic plate where the periodicity presented by masses on the edge of the plate and where the masses reside within the interior of the plate were studied by Hull in this paper. The solution of an elastic plate harmonically loaded in space and time containing periodic edge masses at a time and embedded masses at another time has been derived and compared favorably with previously developed thin plate models at low frequencies. Also a numerical and analytical model of a novel design of a sandwich plate based on the antagonistic approach, and an application of SMA elements, were presented [10]. A two-dimensional model of the flexure of a thin plate reinforced with periodic families of separated thin rods, symmetrical about the middle plane, was analyzed [11].

When periodic analysis is mentioned, it is to point towards investigating the vibration attenuation resulting from periodicity. In the present work, the existence of stop or attenuated bands of frequency of some particular selected periodic plates (see Figure (1)) has been investigated using the finite element method with respect to a reference plain plate by measuring the input-output transfer function at the corners of each periodic plate and comparing it with that of the plain plate experimentally.

\section{NUMERICAL RESULTS}

A 144-DOF finite element model was used to model all the periodic plate cases shown in Fig. 1. The cell of interest was assembled of four elements of the same dimensions except for the "element thickness". Figure 2 presents a sketch of the cell of the plain plate that is used as a reference for the response of the different cases. The main axes of the cell are along the lines joining points 1 and 2, x-axis, and the line joining points 1 and $4, y$-axis. The propagation angle is measured from the $x$-axis counter clockwise. In the plain plate there are no stop "attenuation" bands because there is no change (discontinuity) in the cell geometry. 
Figure 3 presents a sketch of the cell of the periodic plate case \#1. Element \#1 of the cell "thick element" is of thickness $2 \mathrm{~mm}$ while the other elements are of thickness 1 $\mathrm{mm}$. The propagation curves obtained for this case using the same methods introduced for plain plate are presented in Fig. 4. The propagation curves for all cases are obtained at the three main directions at angles $0^{\circ}, 45^{\circ}$, and $90^{\circ}$ to give useful observation. The most significant difference between plain plate and periodic plate case \#1 is the existence of "gaps" between the surfaces indicating the presence of "stop bands".

Case \#2 was designed to confine the wave propagating in the plate in both directions. The geometry was selected to present geometric periodicity in a radial direction from the source point. This configuration is not "repeated" in the $\mathrm{x}$ and $\mathrm{y}$ directions; rather, it expands in a radial-like direction. This configuration follows the one presented by Ref. [9] . The cell shape of this case is presented in Fig. 5, where element 1 is the thin element of thickness $1 \mathrm{~mm}$ and the other elements are of thickness $2 \mathrm{~mm}$.

As expected, the new geometry possesses periodic characteristics that are favorable compared to those of the previous two configurations. Those characteristics can be seen in the shapes of the propagation curves presented in Fig. 6. This cell shape has geometric symmetry between nodes 2 and 4 which results in a corresponding response.

Figure 6 shows bands of attenuation resulting from the change of plate thickness. It also shows the similarity between Figures $6 \mathrm{a}$ and $6 \mathrm{c}$ and between figures $6 \mathrm{~b}$ and $6 \mathrm{~d}$ resulting from the plate symmetry in this direction as they represent the response at nodes 2 and 4 in the cell. For periodic plate case \#3 the cell shape is as presented in Fig. 7. Elements 1 and 3 are of thickness $2 \mathrm{~mm}$ while elements 2 and 4 are of thickness $1 \mathrm{~mm}$.

Figure 8 presents the propagation curves for plate case \#3 obtained by using the same method used for plain plate and plate case \#1. Figures $8 \mathrm{a}$ and $8 \mathrm{~b}$ present the response at node 4 in the cell. It has no geometry change so there is no stop band in the response. On the other hand, the response at nodes 2 and 3 have stop bands as shown in figures $8 \mathrm{c}, 8 \mathrm{~d}, 8 \mathrm{e}$ and $8 \mathrm{f}$, respectively.

\section{EXPERIMENTAL SETUP}

Four plates with different periodic patterns were chosen to investigate their characteristics. They are shown in Fig. 1. The basic geometry of the plates is $40 \times 40 \times 0.2 \mathrm{~cm}$ that is fictitiously divided into smaller $5 \times 5 \mathrm{~cm}$ squares. The plates are made of Aluminum alloy 1050 with modulus of elasticity of $73 \mathrm{GPa}$ and mass density of $2710 \mathrm{Kg} / \mathrm{m}^{3}$. The periodicity is achieved by changing the plates' thicknesses in three different patterns as shown in Fig. 1. All the plates were suspended by flexible wires from point (1) to simulate the free boundary conditions. All the plates were excited at the same point as shown in Fig. 9 using a permanent magnet vibration exciter. The excitation input was measured using force transducer and the responses were measured using four accelerometers at the four corners as shown in fig. 9. The exciter produced a swept sine excitation signal whose frequency 
sweeps the frequency range from 0 to $3.2 \mathrm{KHz}$. This excitation signal is deterministic (nonrandom) and hence it can be repeated several times. This removes the leakage from the measurements and greatly reduces the experiment time since averaging becomes not necessary. Measurements were performed using Brüel \& Kjær multichannel Analysis System type 3550.

The input-output transfer function (TF) is defined as:

$$
\frac{x_{o}(f)}{X_{i}(f)}
$$

where $X_{i}(f)$ and $X_{0}(f)$ are the frequency spectrum of the responses (usually acceleration) at the input and output locations respectively. For each plate of Fig. 1, three TF's were measured at corners 2, 3 and 4 with respect to corner 1 . These corners are shown in Figs. 1a, 1b, 1c and 1d. These TF's were measured and are plotted in Figs. 10, 11 and 12 for the plates of Figs. 1b, 1c and 1d, respectively, as compared to the respective TF's of the plain plate of Fig. $1 \mathrm{a}$.

\section{EXPERIMENTAL RESULTS AND DISCUSSION}

Figure 10 shows the effect of periodicity on attenuating the vibration of the periodic plate case \#1.This figure shows the TF's at the corners 4,2 and 3 with respect to corner 1. As seen in Figure 10-a, significant attenuation bands occurred in the frequency bands [375,470], [1040, 1120], [1430, 1580] and [2300, 2400] Hz, with about $10 \mathrm{~dB}$ attenuation. For Figure 10-b, attenuation of about $10 \mathrm{~dB}$ is observed in the frequency bands $[380,525],[1050,1140]$ and $[1240,1400] \mathrm{Hz}$. Due to symmetry of the plate of Figure 1-b, corner 3 is similar to corner 4, hence it's TF is supposed to be similar. The little difference between Figure 10-b and Figure 10-c may be a result of the inaccuracy of the experimental setup. These bands of attenuation are in consistence with the stop bands shown in Fig. 4.

Similar to Fig. 10, the TF's at corners 2, 3 and 4 with respect to corner 1 of periodic plate case \#2 are plotted in Figure 11. This figure emphasizes the capability of periodic structures to attenuate the vibration. The most significant attenuations are observed in Figure 11-a giving $9 \mathrm{~dB}$ over the frequency range $[1500,1650] \mathrm{Hz}, 10 \mathrm{~dB}$ over $[1875,2150] \mathrm{Hz}$ and over $[2565,2665] \mathrm{Hz}$. For Figure 11-b, attenuation of about $10 \mathrm{~dB}$ over the ranges [425, 535], [720,840], [900, 1020] and [1250, 1350] Hz, $18 \mathrm{~dB}$ over the ranges [1550, 1650], [1725, 1810], and $[2475,2610] \mathrm{Hz}$ are observed. Due to similarity of the plate of Figure 1-c, corner 3 is similar to corner 2, and also the little difference between their transfer functions is due to experimental setup inaccuracies. Some of these attenuated bands shown in Figure 11 are in consistence with the stop bands shown in Fig. 6.

Similarly Figure 12 shows the TF's at corners 4, 2 and 3 with respect to corner 1 of the plate of Figure 1-d respectively. This figure shows that the significant bands of attenuation occur in the frequency band $[1000,1120] \mathrm{Hz}$ with an attenuation of $12 \mathrm{~dB}$ and $[2000,2150] \mathrm{Hz}$ with an attenuation of $14 \mathrm{~dB}$. In Figure 12-b an attenuation of about $9 \mathrm{~dB}$ is observed in the frequency band $[600,700] \mathrm{Hz}$ and attenuation of about $13 \mathrm{~dB}$ in the band $[950,1150] \mathrm{Hz}$. There are less significant bands of attenuation 
shown in figures. It is necessary to remember that the reference plain plate of Figure $1-a$ is thicker, which mean that the plain plate is stiffer than the periodic plates.

From the previous study the comparison between numerical and experimental results can be presented in Table 1.

Table 1. Numerical and experimental attenuation bands.

\begin{tabular}{|c|c|c|c|}
\hline & \multirow{3}{*}{$\begin{array}{c}\begin{array}{c}\text { Numerical attenuation } \\
\text { bands }\end{array} \\
{[250: 300],[1100: 1200],} \\
{[1300: 1600], \text { and }[1900: 2250]}\end{array}$} & \multirow{3}{*}{\begin{tabular}{|l|}
$\begin{array}{c}\text { Experimental attenuation } \\
\text { bands }\end{array}$ \\
{$[250: 500],[1100: 1200]$,} \\
{$[1400: 1600],[1900: 2100]$, and } \\
{$[2300: 2550]$}
\end{tabular}} \\
\hline & & & \\
\hline \multirow{3}{*}{$\begin{array}{l}\text { Periodic } \\
\text { plate case \#1 }\end{array}$} & Node 2 & & \\
\hline & Node 3 & $\begin{array}{l}\text { [450:500], [1100:1400], } \\
{[1900: 2050], \text { and }[2550: 2650]}\end{array}$ & $\begin{array}{l}{[400: 500],[1050: 1150],} \\
{[1250: 1400], \text { and }[2500: 2600]}\end{array}$ \\
\hline & Node 4 & \multicolumn{2}{|c|}{ Same as node 2 due to geometrical symmetry } \\
\hline \multirow[t]{3}{*}{$\begin{array}{l}\text { Periodic } \\
\text { plate case \#2 }\end{array}$} & Node 2 & [1500:2100], and [2800:3100] & $\begin{array}{l}{[200: 600],[750: 850],[950: 1650],} \\
{[1750: 1800],[1850: 2150],} \\
{[2350: 2400],[2450: 2520],} \\
{[2550: 2650],[2750: 2850], \text { and }} \\
{[2900: 3000]}\end{array}$ \\
\hline & Node 3 & {$[1500: 1800]$, and $[2750: 3050]$} & $\begin{array}{l}\text { [400:500], [850:1400], } \\
{[1500: 1650],[1700: 1800],} \\
{[2490: 2600], \text { and }[2780: 2850]}\end{array}$ \\
\hline & Node 4 & \multicolumn{2}{|c|}{ Same as node 2 due to geometrical symmetry } \\
\hline \multirow{3}{*}{$\begin{array}{l}\text { Periodic } \\
\text { plate case \#3 }\end{array}$} & Node 2 & \multicolumn{2}{|c|}{ No attenuation bands resulting from continuous geometry } \\
\hline & Node 3 & [1100:1600], and [2400:2600] & {$[600: 700]$, and [950:1200] } \\
\hline & Node 4 & $\begin{array}{l}{[300: 400],[1050: 1550], \text { and }} \\
{[2850: 3200]}\end{array}$ & $\begin{array}{l}{[250: 500],[1000: 1650], \text { and }} \\
{[1850: 2150]}\end{array}$ \\
\hline
\end{tabular}

\section{CONCLUSION}

Three different patterns of periodic plates with the same size and material were used to study numerically and experimentally the characteristics of periodic plates and their ability to attenuate vibration. Their attenuation with respect to a plain plate was investigated experimentally at different frequency bands. The results obtained show the capability of the numerical model to predict the performance of the periodic plate as well as the capability of the periodic plates in attenuating vibration in certain frequency bands.

\section{REFERENCES}

[1] Tsung-Tsong Wu, Zi-Gui Tsai and Tzung-Chen Wu, 'Evidence of complete band gap and resonances in a plate with periodic stubbed surface', Taiwan : APPLIED PHYSICS LETTERS 93, 2008. 
[2] F. Casadei, M. Ruzzene, L. Dozio and K. A. Cunefare, 'Broadband vibration control through periodic arrays of resonanat shunts: experimental investigation on plates', Smart Materials and Structures, stacks.iop.org/SMS/19/015002. [Online] November 19, 2009.

[3] Abo Elsooud and Mohammad Tawfik, 'Vibration control of plates using periodically disributed shunted piezoelectric patches', University of Maryland : S.N., 2003.

[4] Periodic shunted arrayes for the control of noise radiation in an enclosure. Filippo Casadei, Lorenzo Dozio, Massimo Ruzzene and Kenneth A. Cunefare. Journal of Sound and Vibration, p. 15.2010.

[5] Yeung, Yum-Tong Siu and Sai-Kee. A generalized Bloch's theorem and the hyperbolicity of the complement of an ample divisor in an Abelian variety. USA : Mathematics Subject Classification, 1991.

[6] Diperna, Daniel T/ Blake, William K/ and Diperna, Xingguang Z. Computational of the homogeneous and forced solutions of a finite length, line-driven, submerged plate. The Journal of the Acoustical Society of America. 2006.

[7] W. Xiao, G.W. zeng and Y. S. Cheng. Flexural vibration band gaps in a thin plate containing a periodic array of hemmed discs, ELSEVIER, p. 7.2006.

[8] J. B. Casimir, S. Kevorkian, T. Vinh.. J.B. Casimir, S. Kevorkian and T. Vinh.The dynamic stiffness matrix of two-dimensional elements: application to Kirchhoff's plate continuous elementsFrance: Journal of Sound and Vibration , 2005.

[9] Hull, Andrew J. Dynamic response of an elastic plate containing periodic masses., ELSEVIER, p. 20.2007.

[10] O. A. Ganilova and M.P.Cartmell. An analytical model for the vibration of a composite plate containing an embedded periodic shape memory alloy structure. Elsevier, 2009.

[11] S.A Nazarov, G.H.Sweers and A.S.Slutskii. The flexural rigidity of a thin plate reinforced with periodic systems of separated rods. Journal of Applied Mathematics and Mechanics, p. 10. 2008. 


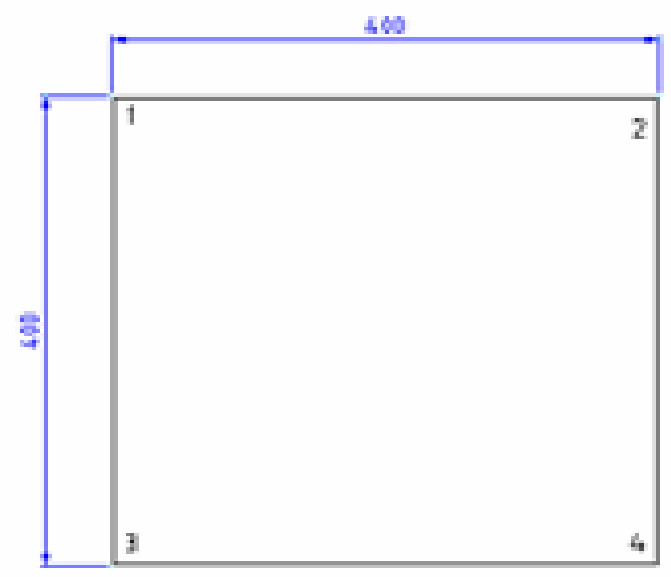

[a] Plain plate

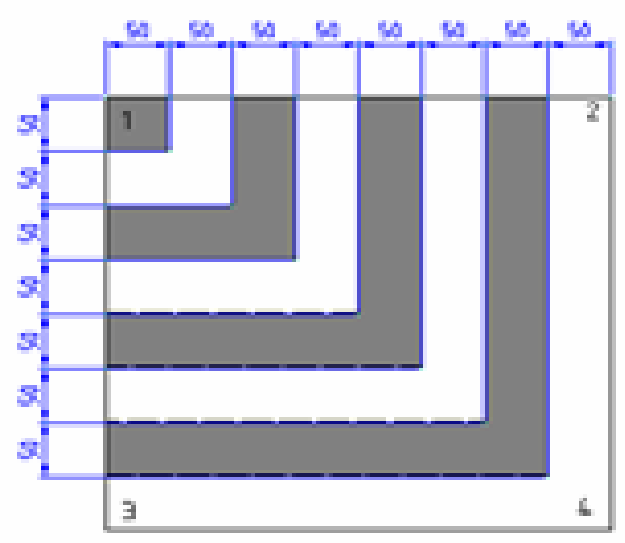

(k) Periodic plete cose $|z|$

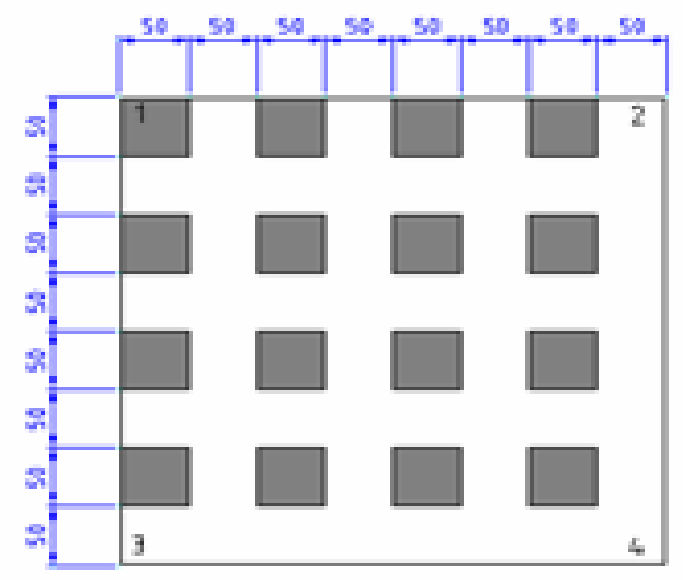

[p] Periodic plete cese [1]

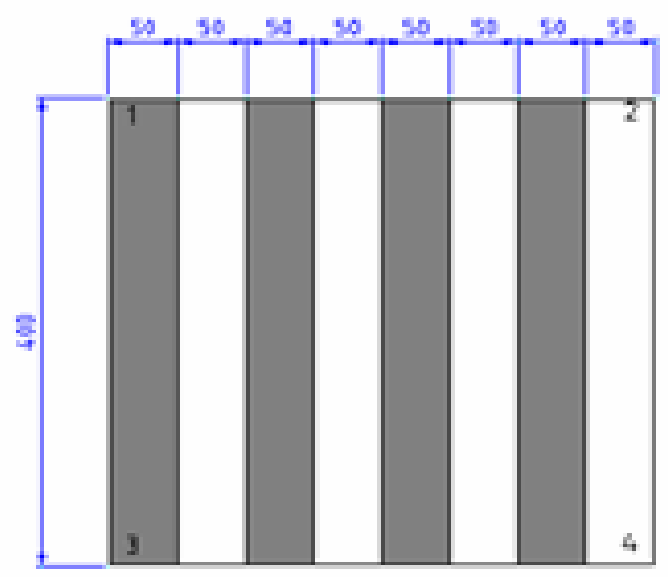

[d] Feriodic Plete cose |B]

Fig. 1. The different four cases for the tested plates.

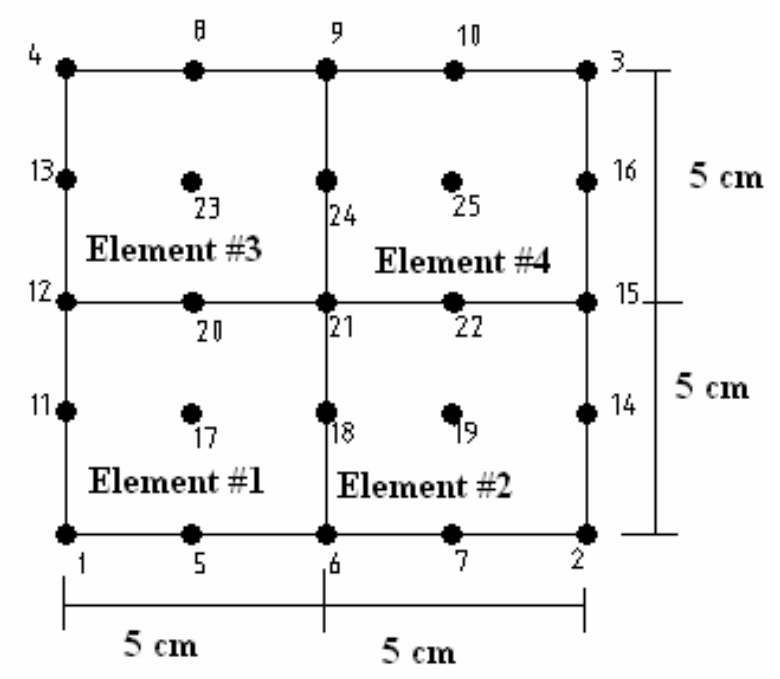

Fig. 2. A sketch of a single cell of plain plate 


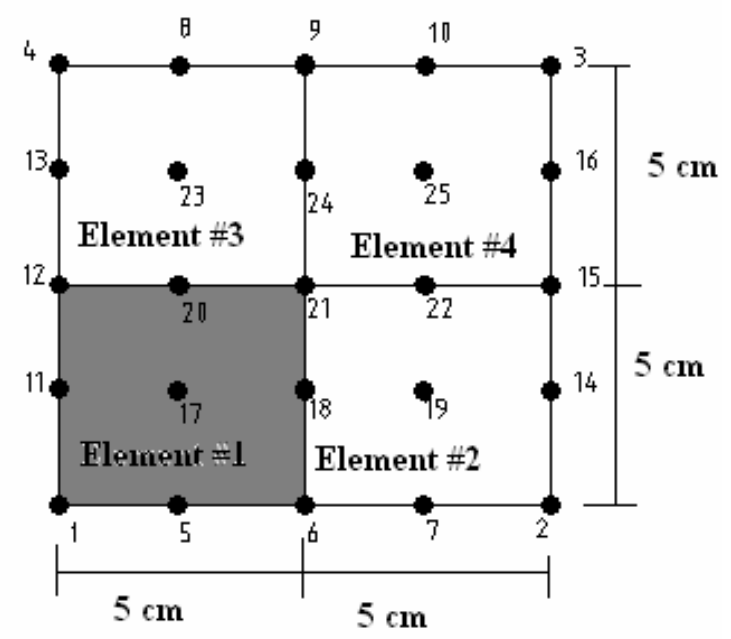

Fig. 3. A sketch of a single cell of plate Case\#1.

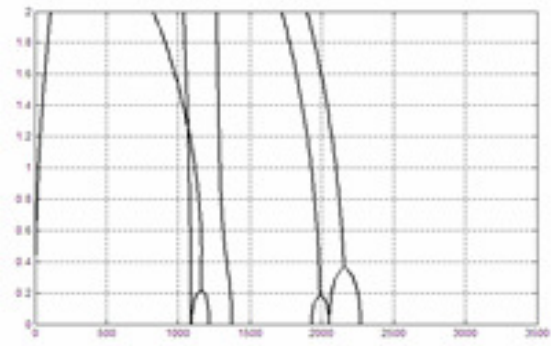

(a)

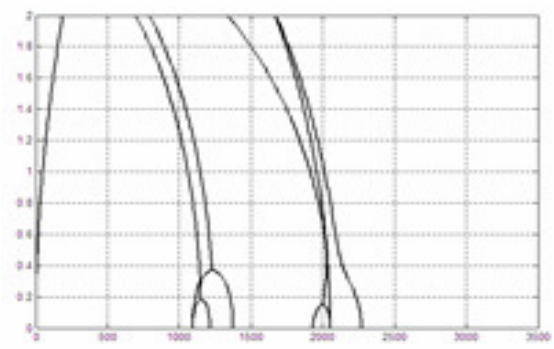

(c)

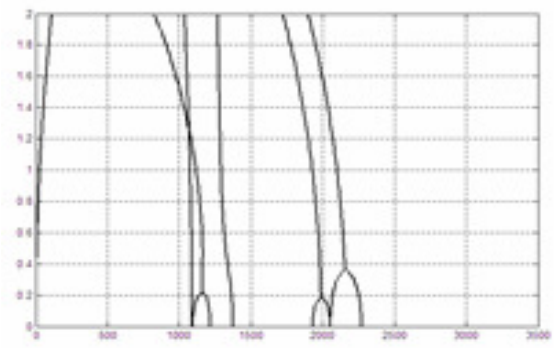

(e)

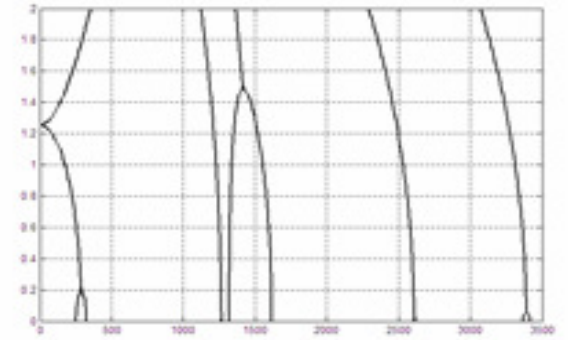

(b)

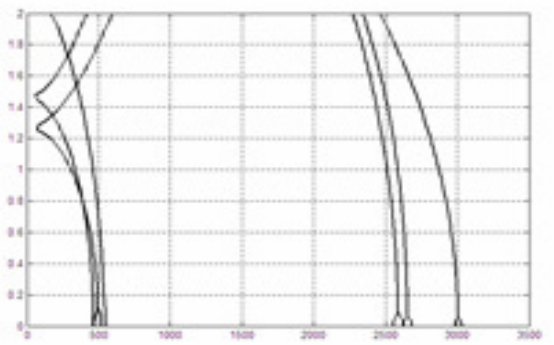

(d)

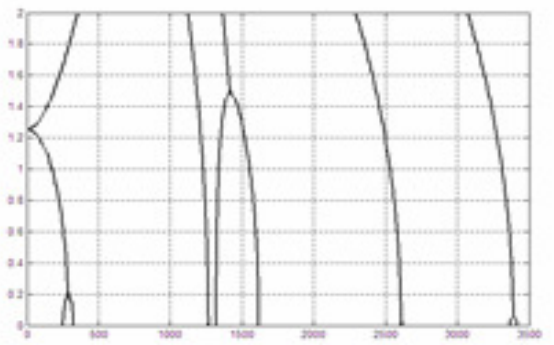

(f)

Fig. 4. Propagation curves of plate case \#1 where $(a) \mu_{x}=0 \& \mu_{y}=\alpha+0(b) \mu_{x}=0 \& \mu_{y}=\alpha+i \beta$ (c) $\mu_{y}=\mu_{x}=\alpha+0$ (d) $\mu_{y}=\mu_{x}=\alpha+i \beta(e) \mu_{y}=0 \approx \mu_{x}=\alpha+0$ (f) $\mu_{y}=0 \& \mu_{x}=\alpha+i \beta$. 


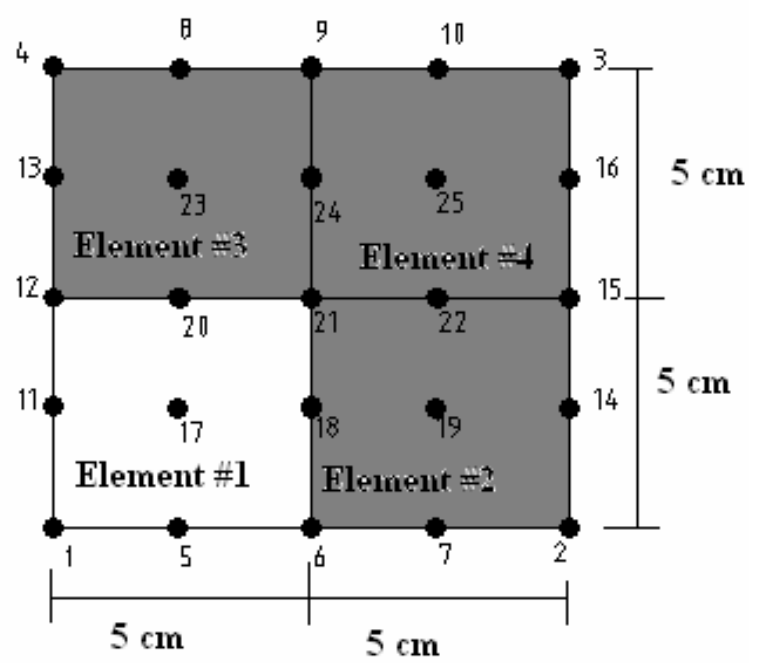

Fig. 5. A sketch of a single cell of plate Case\#2.

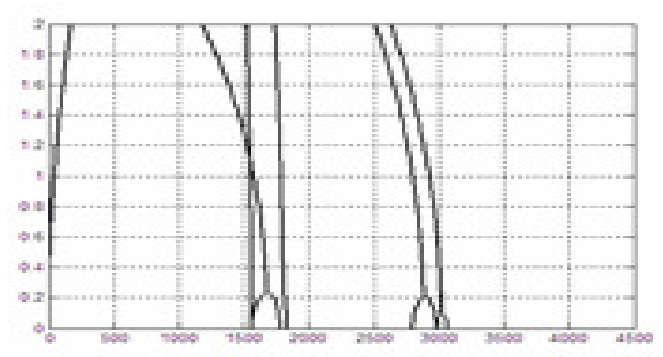

(a)

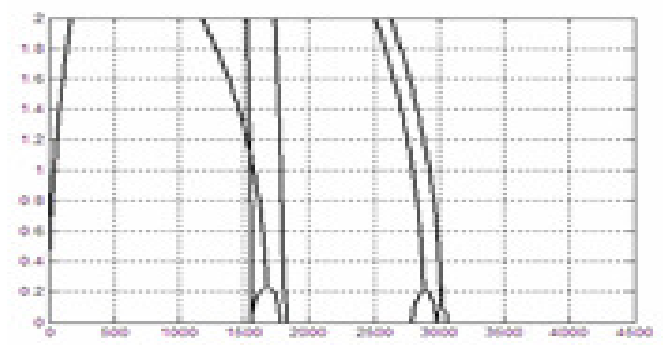

[c)

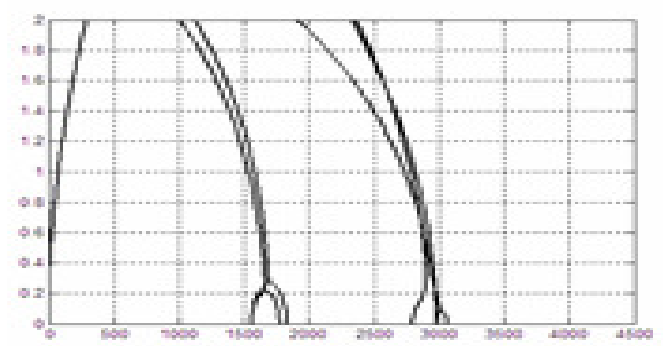

(e)

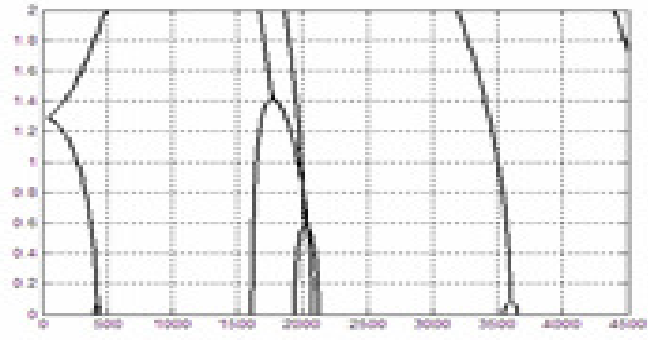

[b]

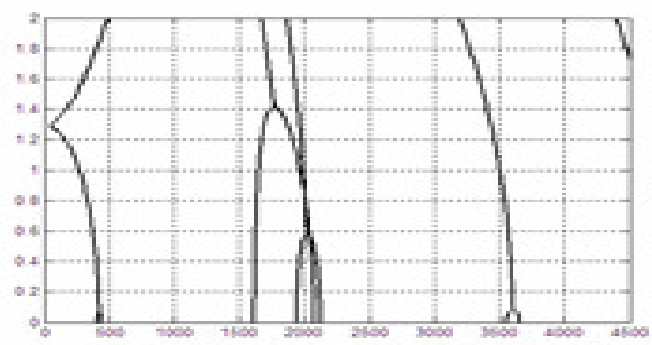

(cl)

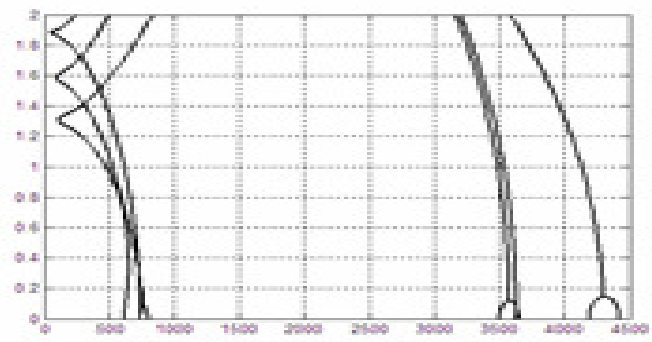

(f)

Fig. 6. Propagation curves of Plate case \#2 where (a) $\mu_{x}=0 \& \mu_{y}=a+0$ (b) $\mu_{x}=0 \delta \mu_{y}=\alpha+i \beta$ (c) $\mu_{y}=0 \delta \mu_{x}=\alpha+0$ (d) $\mu_{y}=0 \delta \mu_{x}=\alpha+i \beta$ (e) $\mu_{y}=\mu_{x}=\alpha+0$ (f) $\mu_{y}=\mu_{x}=\alpha+i \beta$ 


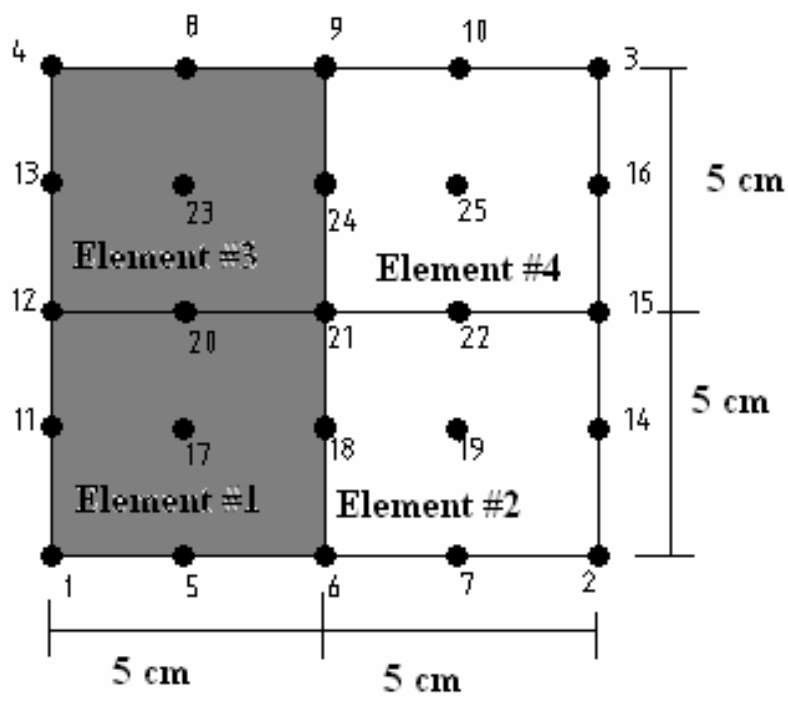

Fig. 7. A sketch of a single cell of plate Case\#3.

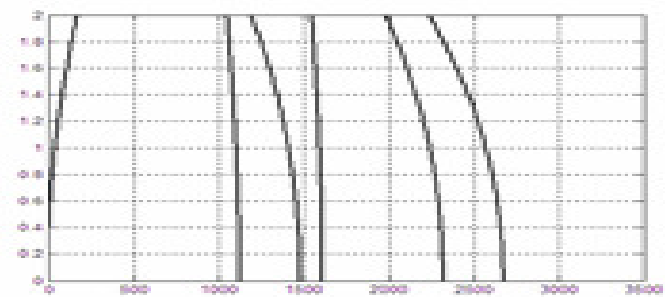

(a)

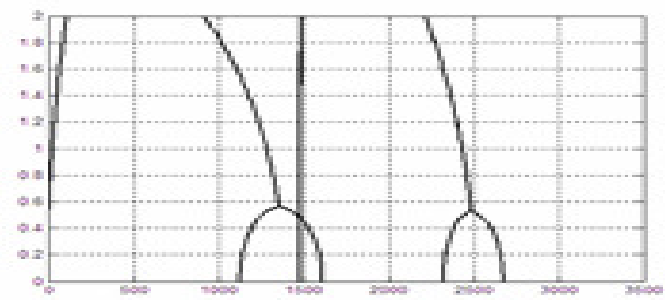

[c]

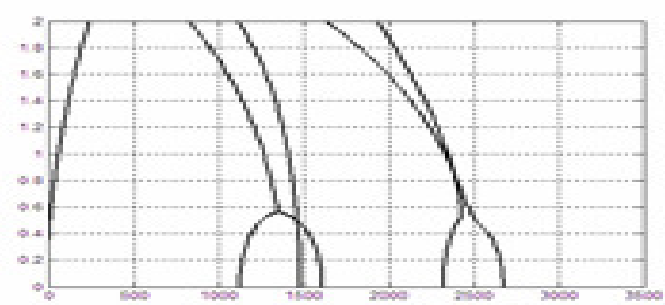

(e)

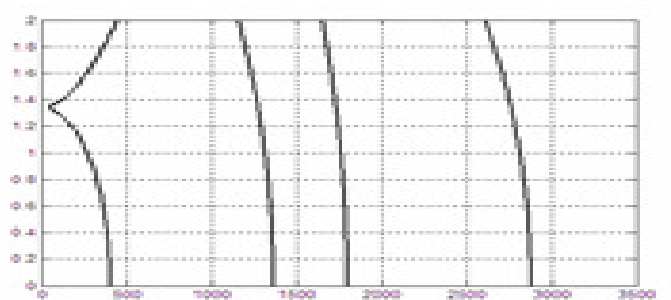

(b)

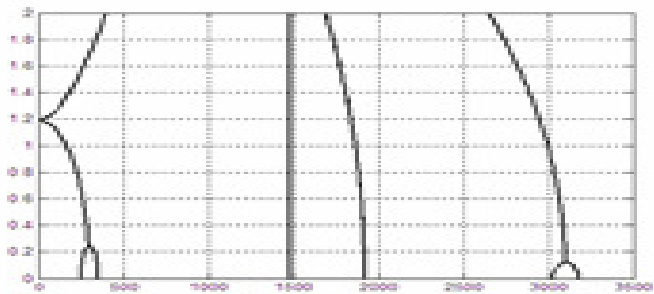

(cl)

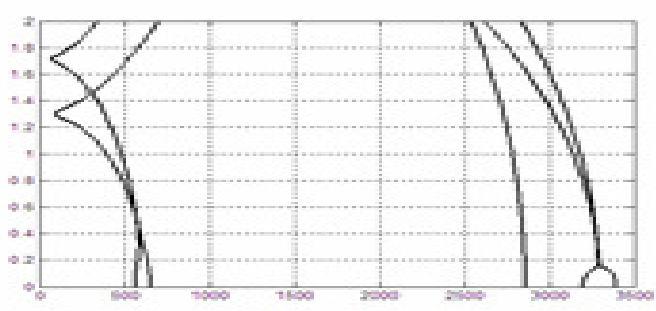

(f)

Fig. 8. Propagation lines of plate case \#3 where (a) $\mu_{x}=0 \& \mu_{y}=\alpha+0$ (b) $\mu_{x}=0 \& \mu_{y}=\alpha+i \beta$ (c) $\mu_{y}=0 \delta \mu_{x}=\alpha+0$ (d) $\mu_{y}=0 \& \mu_{x}=\alpha+i \beta(e) \mu_{y}=\mu_{x}=\alpha+0$ and (f) $\mu_{y}=\mu_{x}=\alpha+i \beta$. 

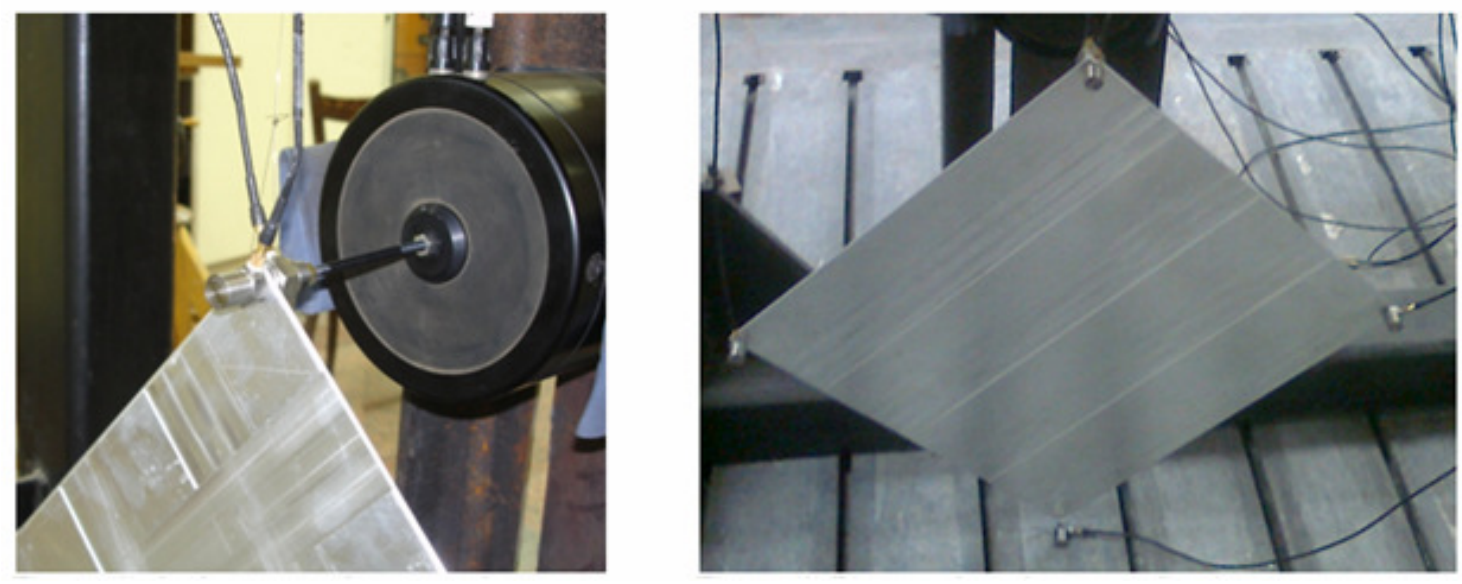

Fig. 9. The force transducer attachment to periodic plate case \#2 and accelerometer fixation at the corners of periodic plate case \#3.

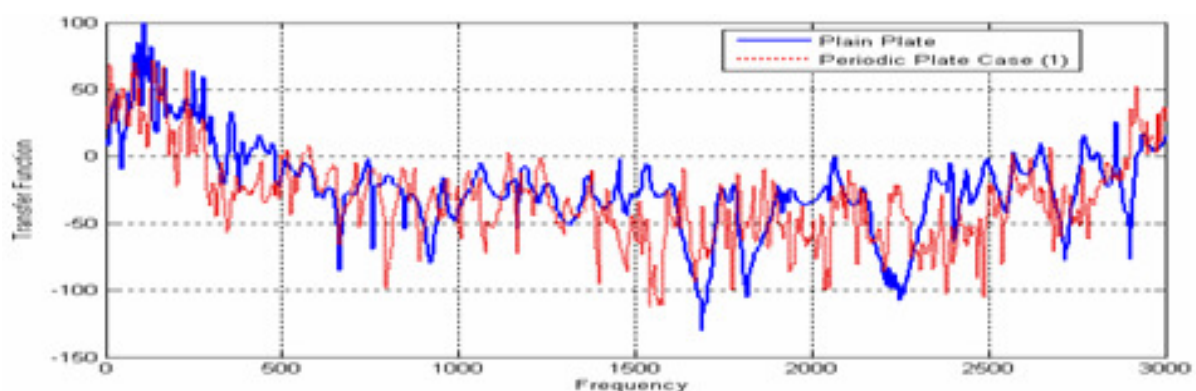

(a)

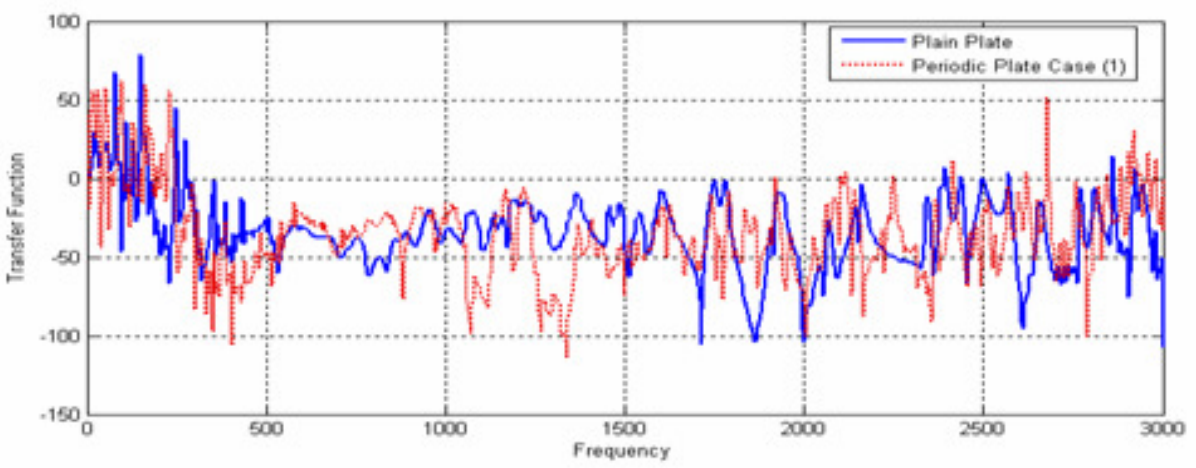

(b)

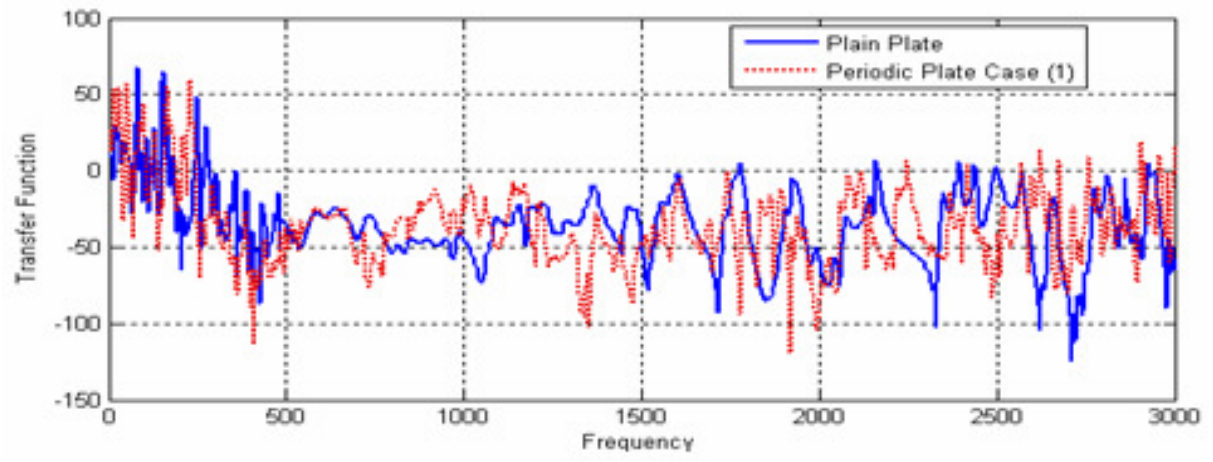

(c)

Fig. 10. Transfer function of the periodic plate of Fig.1-b as compared to the plain plate of Fig. 1-a. 


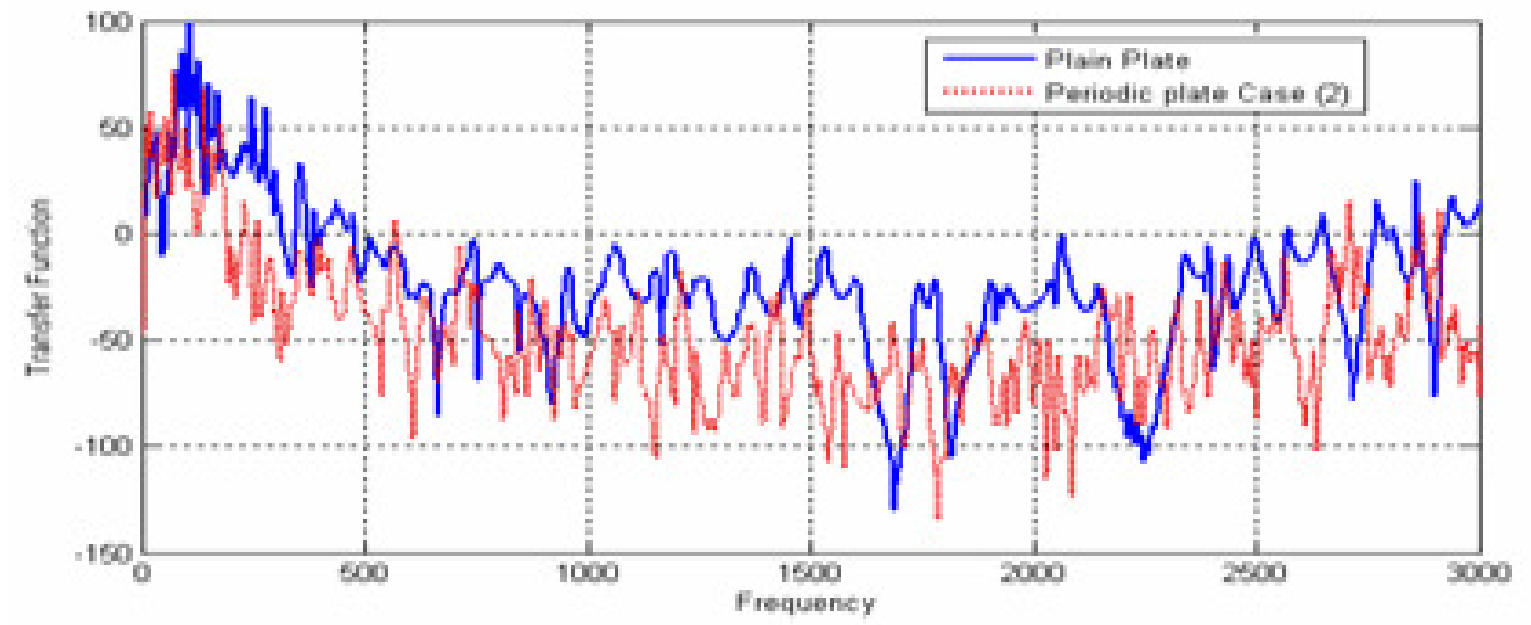

[s]

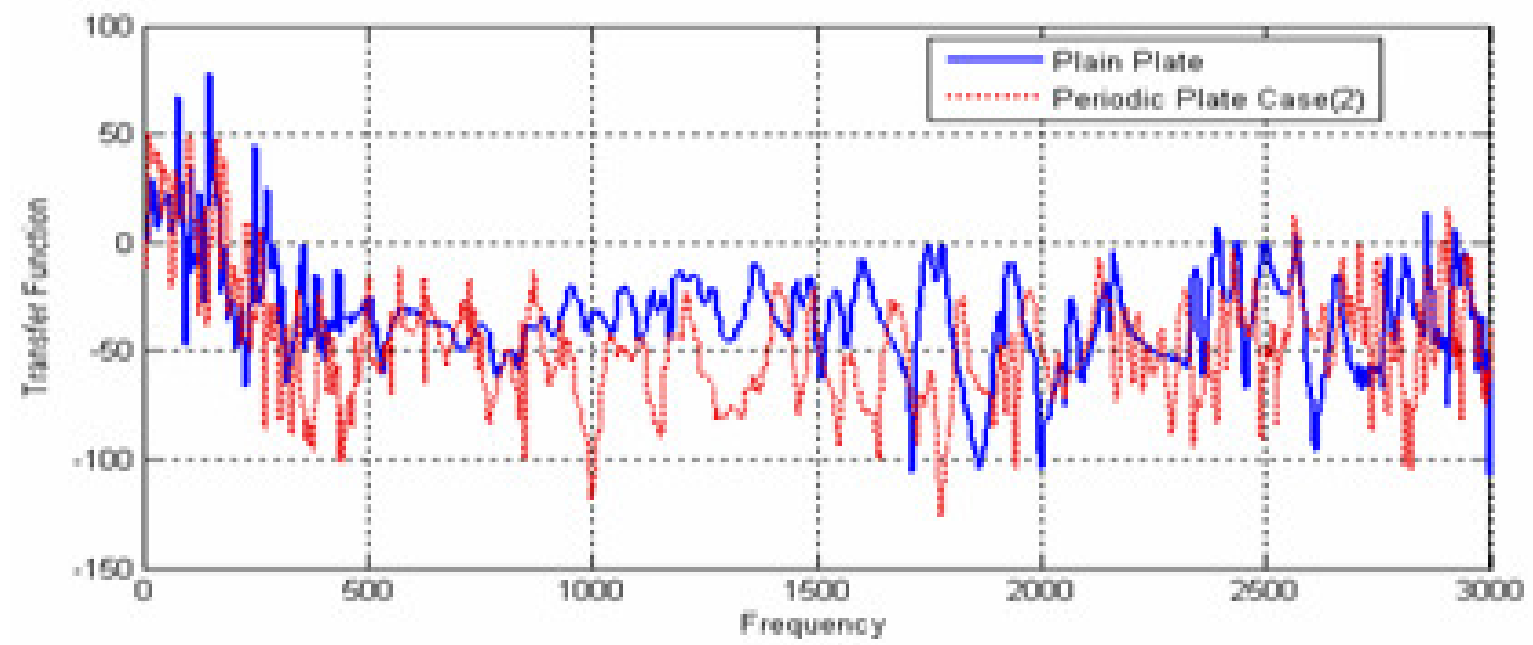

(b)

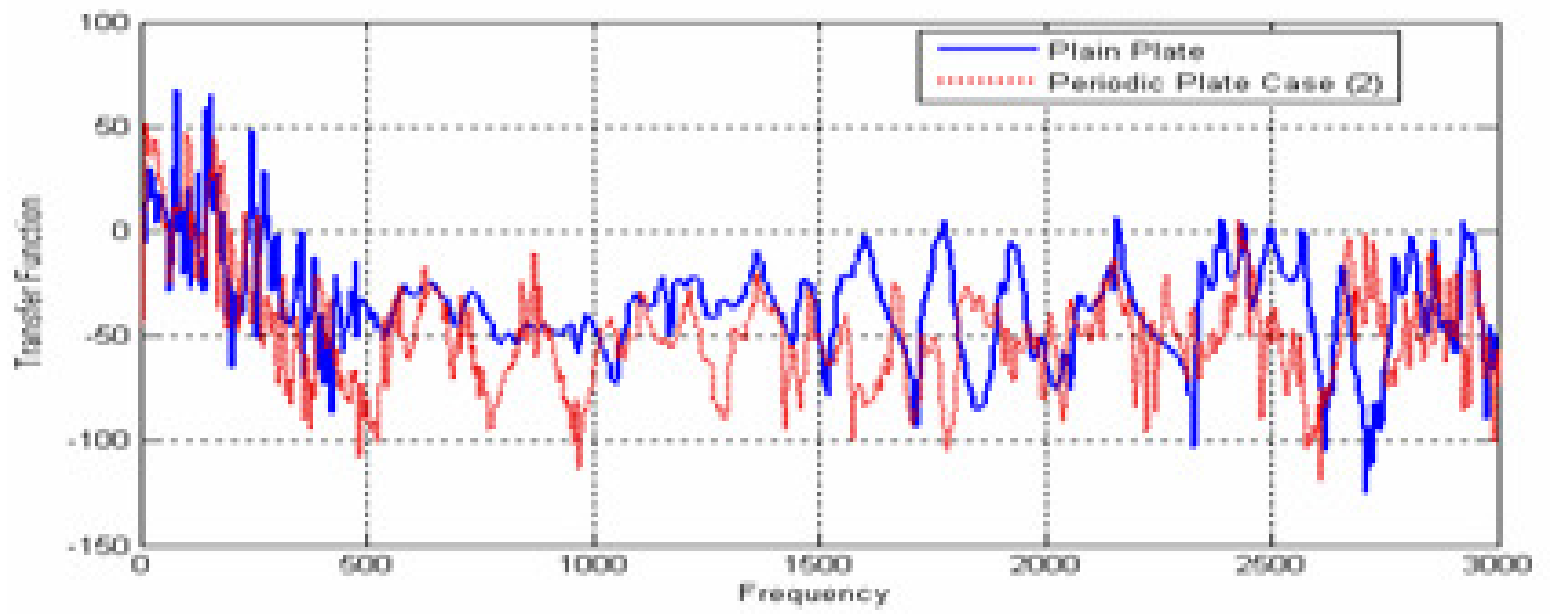

[c]

Fig. 11. Transfer function of the periodic plate of Fig. 1-c as compared to the plain plate of Fig. 1-a. 


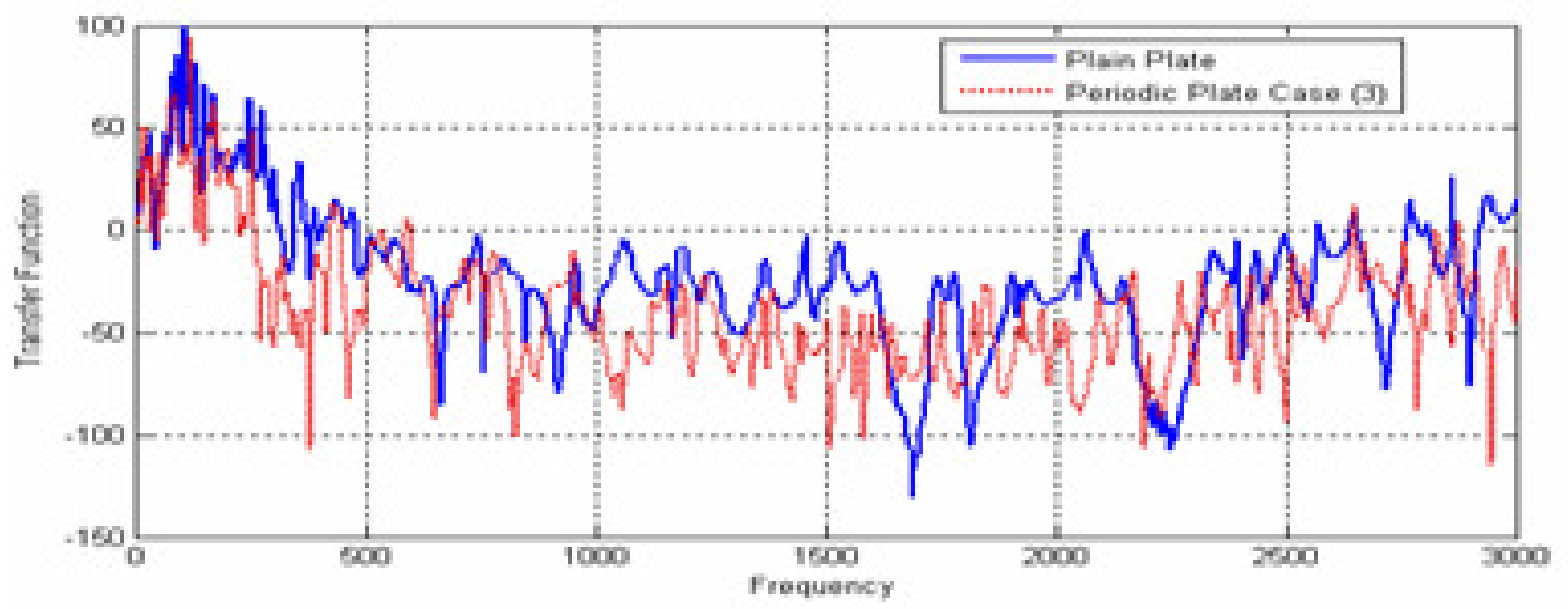

[a]

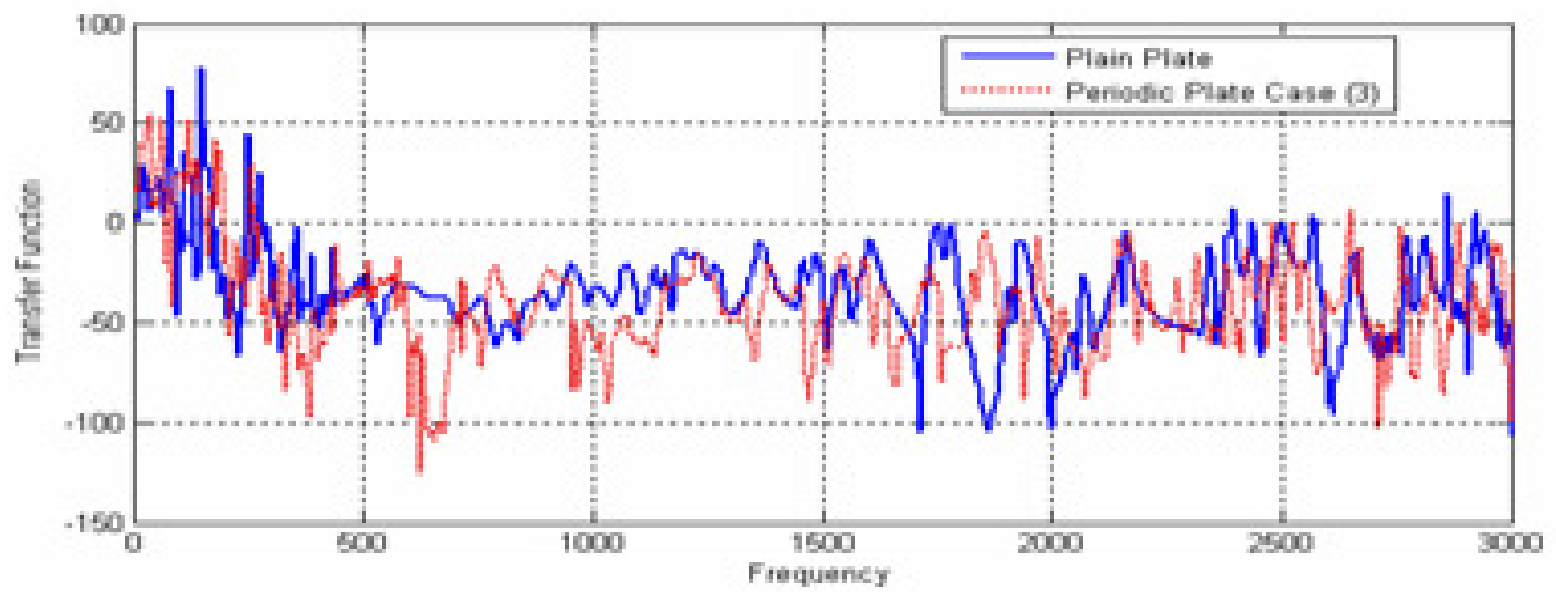

[b]

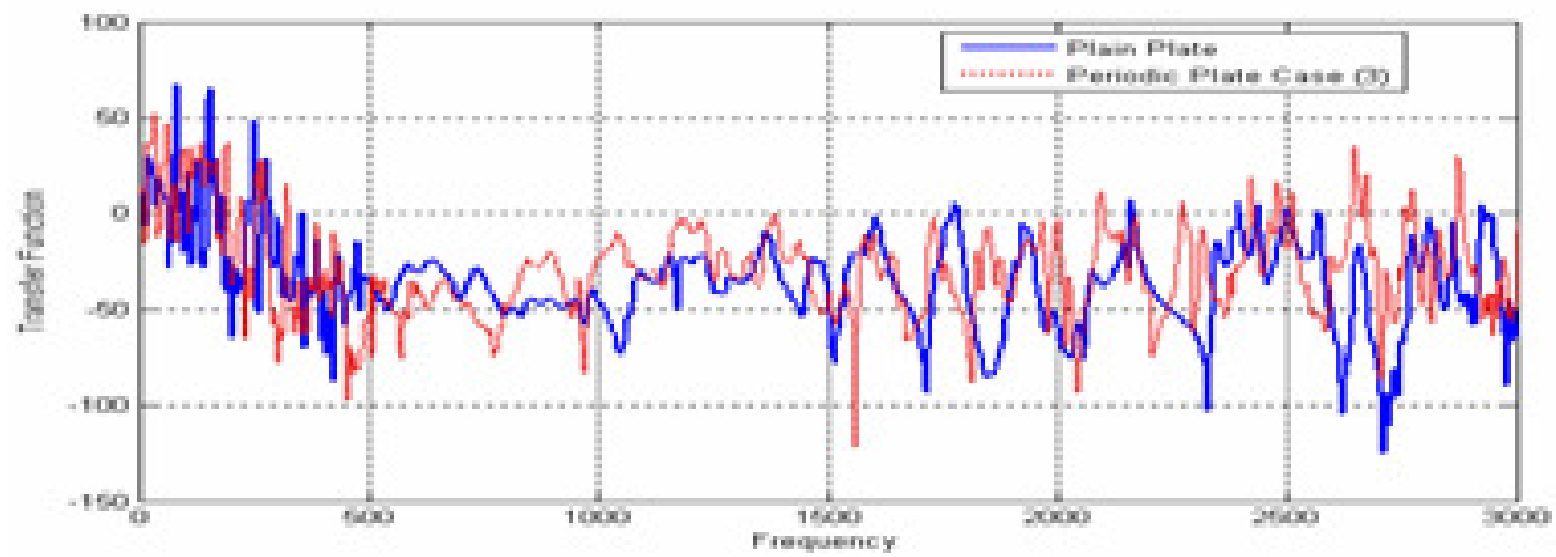

$(c)$

Fig. 12. Transfer function of the periodic plate of figure 1-d as compared to the plain plate of figure 1-a. 